\section{Plastic modification of the cornea by pneumatic force corrects myopia: Pneumatic keratology \\ A Medina ${ }^{1}$}

\begin{abstract}
Purpose The eyeball and, in particular, the cornea deform in vitro by the application of a distending force like other visco-elastic tissue. If the force is large enough, the cornea strains beyond the elastic range, and a permanent deformation occurs. Such permanent strain is referred to as 'plastic' strain. The phenomenon, however, has never been observed or produced on living tissue. This report seeks to demonstrate that the central radius of a patient's cornea can be altered in a controlled manner designed to correct refractive errors.

force to strips of cornea held between two grips. Corneal biomechanical properties have also been measured by pressurizing the cornea within an artificial anterior chamber that uses internal pressure as the stress and by measuring the strain by means of marks on the cornea (bulge testing method). ${ }^{6-11}$ Biomechanical measurements of excised donor human corneas report a plastic deformation of the cornea in vitro. 5,12 No attempt has been made until now to plastically deform the human cornea in vivo or to alter the collagen fibers of the stroma for corrective purposes.
\end{abstract} Methods To plastically deform the living cornea, we applied a vacuum to the cornea of eight rabbits and five human eyes with a novel device. This device consists of a chamber of $11 \mathrm{~mm}$ in diameter. The chamber is radially divided into four interconnected sub-chambers. Results Here we show that a strain can be achieved in vivo with a force produced by the application of the specially designed chamber where air is evacuated. An anatomical modification of the cornea of humans and rabbits was achieved. The deformation of the cornea was plastic, and therefore permanent. Conclusions The method described herePneumatic Keratology- can be used to alter the cornea by non-invasive means. A vacuum chamber with radial openings alters the collagen fibers in the stroma and flattens the cornea. A flatter cornea corrects or reduces myopia.

Eye (2017) 31, 1621-1627; doi:10.1038/eye.2017.123; published online 16 June 2017

\section{Introduction}

Several methods have been used to elucidate the corneal tissue biomechanics. The tensile strip extensiometry, ${ }^{1-5}$ involves applying a tensile

\section{Materials and methods}

All procedures performed in studies involving human participants were in accordance with the ethical standards of the institutional review board of the Hospital de la Cruz Roja in Barcelona and with the 1964 Helsinki declaration and its later amendments or comparable ethical standards. All applicable international, national, and/or institutional guidelines for the care and use of animals were followed.

Informed consent was obtained from all individual participants included in the study. Additional informed consent was obtained from all individual participants for whom identifying information is included in this article.

To plastically deform the living cornea we applied a vacuum to the cornea of eight rabbits and five human eyes with a novel device. The number of rabbits was selected based on accepted sample size calculators. The rabbits, three female and five male, were randomly selected from an albino colony. The small sample size was considered sufficient because a substantial effect was expected. The rabbits were 7 to 10 weeks old at the time of treatment. The novel device consists of a chamber of $11 \mathrm{~mm}$ in diameter. The chamber is radially divided in four interconnected sub-chambers. One side of
Massachusetts Institute Of Technology EE Research Laboratory, Cambridge, MA, USA

Correspondence:

A Medina, Massachusetts Institute Of Technology EE Research Laboratory, Cambridge 02139, MA, USA

Tel: +1 408365 4329;

Fax: +1 8472417740 . E-mail: puerta@alum.mit. edu

${ }^{1}$ Present address: Multivision Research 450 S Abel St. Unit 361361, Milpitas, California 90536, USA.

Received: 5 September 2016 Accepted in revised form: 27 April 2017 Published online: 16 June 2017 
the cylindrical chamber is closed, while the other side of the chamber is open, exposing the sub-chamber openings, Figure 1. When the open side is placed in contact with the cornea, the cornea closes and seals the sub-chamber openings. A vacuum of up to $760 \mathrm{~mm}-\mathrm{Hg}$ was then applied to the chamber, exerting a force on the cornea. A vacuum of $760 \mathrm{~mm}-\mathrm{Hg}$ means that the atmospheric pressure was removed from the front of the cornea, therefore a pressure of $760 \mathrm{~mm} \mathrm{Hg}$ plus the intraocular pressure pushed the cornea outwards at the location of the chambers.

The rabbits were first treated with the device. The chamber was applied to their right eye and vacuum maintained for 5-10 min. The corneal topography of the eyes was measured pre-treatment and 3 days, three months, and 1 year post- treatment with a Placido based topographer, (Atlas 995; Carl Zeiss Meditec, Dublin, CA, USA). All measurements were blind in that they were made without knowledge of the particular rabbit and its prior readings. Pre-treatment and post- treatment interocular differences in central corneal power were compared using the Student's $t$-test of paired comparisons. The left, untreated eye was used as a control. The eyes were examined at the time of the topography when basic parameters of the eyes were also measured. After the conclusion of the one year posttreatment, rabbit 8 was also subjected to the same procedure at a pressure of $3 \mathrm{~atm}$ for $10 \mathrm{~min}$ to acquire further safety information. Rabbit corneas were sent for histological or micro-morphological analysis.

Human subjects were then selected based on the criteria described next. Subjects were 30 to 50 years of age. Their eyes were myopic in a range of 2 to 20 Diopters (D). No ocular disease was present. Only subjects with $1 \mathrm{D}$ or less of astigmatism were included. A total of 5 subjects met

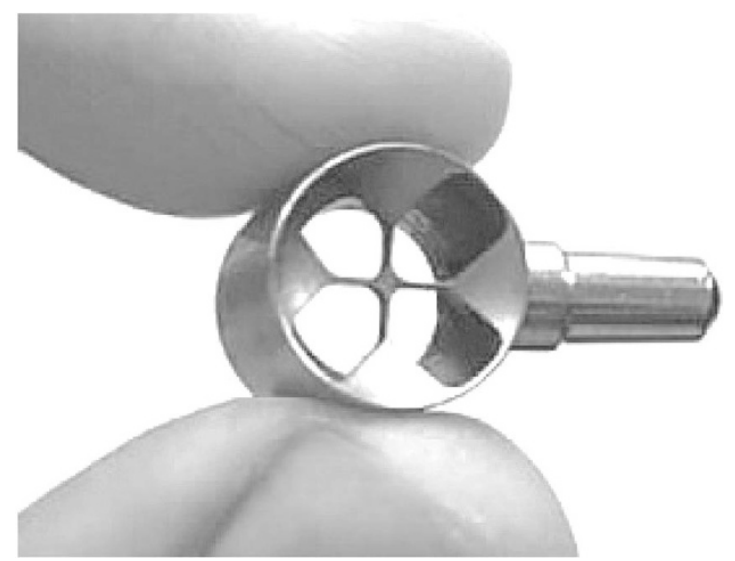

Figure 1 The device used to alter the shape of the cornea consists of a vacuum chamber with openings that produce a force when placed on the cornea.

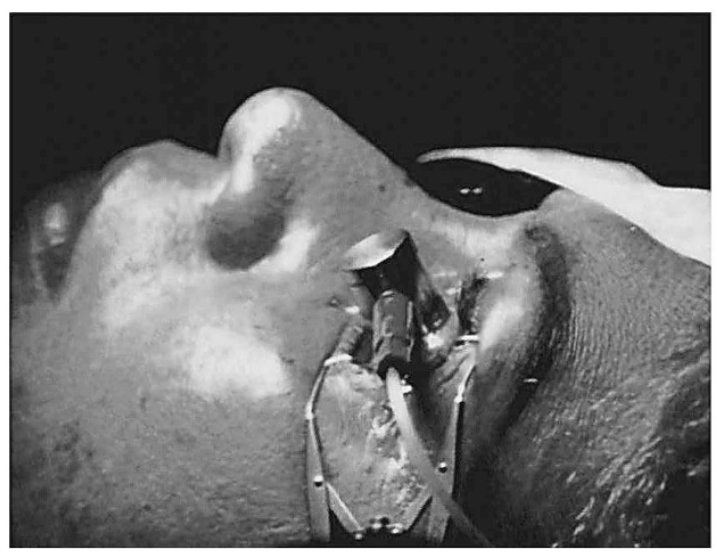

Figure 2 Application of the device to a patient's left eye showing the chamber and vacuum line. The speculum is not necessary, but a convenience.

the above criteria. Subjective refractions were performed by the same experienced refractionist, who had no knowledge of the study. Automated refractions were also used for objective confirmation of the refractive change.

Visual acuity was recorded.

To detect potential safety issues of the procedure, the following exams were performed: Slit lamp exam, recording of Intraocular Pressure (IOP), pupillary reflex, direct and indirect ophthalmoscopy, Optical Coherence Tomography (OCT), and Confocal Laser Scanning Microscopy (CLSM).

We then confirmed, as described in results below, that the application of vacuum was also innocuous on the human eyes by applying vacuum at progressively higher pressures, of up to $760 \mathrm{~mm} \mathrm{Hg}$, and for longer durations, up to a five minute period. Thereafter, we applied a vacuum of $760 \mathrm{~mm} \mathrm{Hg}$ for $5 \mathrm{~min}$ to the five left eyes of the volunteers. All treatments were done at a room temperature of $25^{\circ} \mathrm{C}$ under topical anesthesia. Figure 2 is a photograph of a patient's eye during the procedure.

The refractions, visual acuity tests, and exams except OCT were done pre-treatment and 1 day, 2 months, and 2 years post-treatment. The treatment and examination were completed on each subject's eyes before proceeding to the next subject. Therefore, if an adverse effect was detected, the study could be aborted.

\section{Results}

We report the changes in anatomy observed after the treatment. Corneal topography maps showed that the radius of curvature in the center of the cornea (in a zone of $3 \mathrm{~mm}$ of diameter) increased after treatment and decreased in the periphery. The corneal central radius of the intact rabbit eye was compared with the radius three days after treatment. The three-day change in the radius 
of the treated eye was found statistically significant. Pre-treatment radius of $r=6.50 \mathrm{~mm}$ or $51.92 \mathrm{D}$, standard deviation, $\sigma=0.80 \mathrm{D}$ versus third day post-treatment radius of $r=6.59 \mathrm{~mm}$ or $51.19 \mathrm{D}$, standard deviation, $\sigma=0.67 \mathrm{D}, p=0.006$ (Table 1a). In Tables $1 \mathrm{a}-\mathrm{d}$, radius and power are the mean of the maximum and minimum values in the central cornea. The flattening of the cornea in diopters for each rabbit is shown in bar graphs in Supplementary Figure A. All corneas flattened after the treatment. About half of the corneas flattened about $1 \mathrm{D}$; one flattened about $1.75 \mathrm{D}$ and three flattened about $0.25 \mathrm{D}$. We wanted to evaluate the long-term stability of the change in the radius of curvature without interference from the natural variation of over $10 \mathrm{D}$ that occurs in the corneas of young rabbits. To accomplish that we compared the average radii of the right eye (OD) and left eyes (OS) three months after treatment and one year after treatment. All radii are shown in Table 1b. This comparison uses the non-treated (left) eye as the control. The pre-treatment difference between the average radius of the right eye $(6.50 \mathrm{~mm}$ or $51.87 \mathrm{D})$ and the left eye (6.52 mm or $51.75 \mathrm{D})$ was not statistically significant. The same inter-ocular difference was statistically significant 3 months and 1 year after treatment. The comparison at the third month showed the following statistics: OD $r=7.66 \mathrm{~mm}$ or $44.10 \mathrm{D}, \sigma=0.70 \mathrm{D}$ versus OS $r=7.57 \mathrm{~mm}$ or $44.60 \mathrm{D}, \sigma=0.50 \mathrm{D}, p=0.026$. The comparison at about one year post-treatment showed: OD $r=8.23 \mathrm{~mm}$ or $41.01 \mathrm{D}, \sigma=0.64 \mathrm{D}$ versus OS $r=8.13 \mathrm{~mm}$ or $41.50 \mathrm{D}$, $\sigma=0.68 \mathrm{D}, p=0.015$. Corneal flattening was therefore stable and no significant regression of the effect was observed after three months. The flattening of rabbits' corneas regressed to half a diopter three months posttreatment, and remained stable thereafter. This estimation of refractive change in rabbits is based on the correlation between the power of a corneal optical zone of $3 \mathrm{~mm}$ of

Table 1a Pre-treatment and Post-treatment corneal radii and power (rabbit)

\begin{tabular}{|c|c|c|c|c|c|c|c|}
\hline \multirow{2}{*}{$R A B B I T$} & \multicolumn{4}{|c|}{ Pre-treatment } & \multicolumn{3}{|c|}{3 days post-treatment } \\
\hline & \multicolumn{2}{|c|}{ Radius (mm) } & \multicolumn{2}{|c|}{ Power (D) } & $\frac{\text { Radius }(\mathrm{mm})}{\mathrm{OD}}$ & $\frac{\text { Power }(D)}{\text { OD }}$ & $\frac{\text { Dif. (D) }}{O D}$ \\
\hline No. 1 & 6.56 & 6.57 & 51.50 & 51.37 & 6.58 & 51.25 & 0.25 \\
\hline No. 2 & 6.39 & 6.38 & 52.87 & 52.87 & 6.60 & 51.12 & 1.75 \\
\hline No. 4 & 6.34 & 6.47 & 53.25 & 52.12 & 6.45 & 52.37 & 0.88 \\
\hline No. 5 & 6.63 & 6.56 & 50.87 & 51.50 & 6.70 & 50.37 & 1.13 \\
\hline No. 6 & 6.59 & 6.35 & 51.25 & 53.12 & 6.71 & 50.25 & 1.00 \\
\hline No. 7 & 6.54 & 6.41 & 51.62 & 52.62 & 6.56 & 51.50 & 0.12 \\
\hline No. 8 & 6.5 & 6.52 & 51.87 & 51.75 & 6.55 & 51.50 & 0.37 \\
\hline Average & 6.50 & 6.48 & 51.92 & 52.08 & 6.59 & 51.19 & 0.81 \\
\hline Stdev & 0.099 & 0.091 & 0.8046 & 0.72081 & 0.08348439 & 0.671225 & 0.54068 \\
\hline
\end{tabular}

Table 1b Post-treatment corneal radii and power at three months and a year (rabbit)

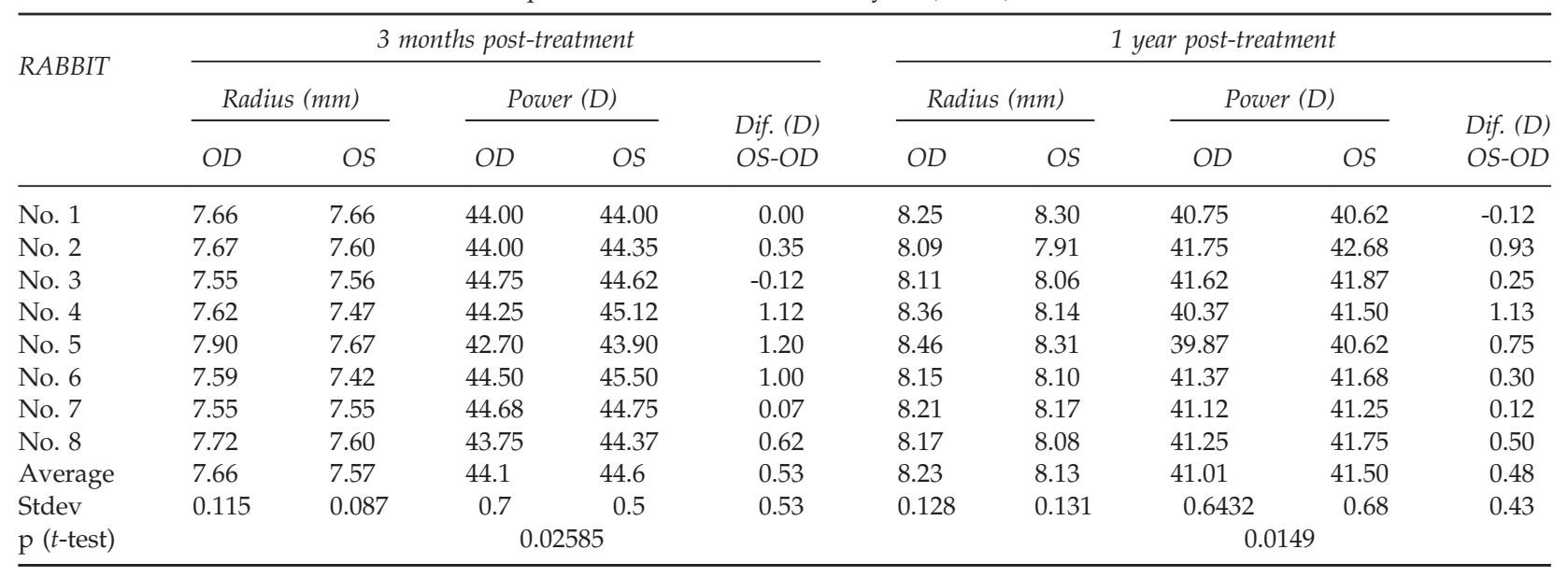


Table 1c Pre-treatment and Post-treatment OS corneal radii, power, refraction and corrected visual acuity (human)

\begin{tabular}{|c|c|c|c|c|c|c|c|c|}
\hline \multirow{2}{*}{ SUBJECT } & \multicolumn{4}{|c|}{ Pre-treatment } & \multicolumn{4}{|c|}{1 day post-treatment } \\
\hline & Radius (mm) & Power (D) & Refraction (D) & $C D V A$ & Radius (mm) & Power (D) & Refraction $(D)$ & $C D V A$ \\
\hline No. 1 & 7.52 & 44.91 & -2.75 & 1.0 & 7.59 & 44.50 & -2.25 & 1.0 \\
\hline No. 2 & 7.83 & 43.12 & -3.00 & 1.5 & 7.99 & 42.30 & -2.12 & 1.5 \\
\hline No. 3 & 7.40 & 45.61 & -6.00 & 1.0 & 7.54 & 44.75 & -5.00 & 1.0 \\
\hline No. 4 & 7.58 & 44.56 & -6.12 & 0.8 & 7.83 & 43.12 & -4.75 & 0.8 \\
\hline No. 5 & 7.71 & 43.80 & -20.00 & 0.3 & 8.55 & 39.50 & -15.00 & 0.5 \\
\hline
\end{tabular}

Table 1d Post-treatment OS corneal radii, power, refraction and corrected visual acuity at two months and two years (human)

\begin{tabular}{|c|c|c|c|c|c|c|c|c|}
\hline \multirow{2}{*}{ SUBJECT } & \multicolumn{4}{|c|}{2 months post-treatment } & \multicolumn{4}{|c|}{2 years post-treatment } \\
\hline & Radius (mm) & Power (D) & Refraction $(D)$ & $C D V A$ & Radius (mm) & Power (D) & Refraction (D) & $C D V A$ \\
\hline No. 1 & 7.61 & 44.38 & -2.25 & 1.0 & 7.58 & 44.50 & -2.00 & 1.0 \\
\hline No. 2 & 8.00 & 42.10 & -2.00 & 2 & 7.94 & 42.50 & -2.25 & 1.6 \\
\hline No. 3 & 7.59 & 44.50 & -4.75 & 1.0 & 7.59 & 44.50 & -5.00 & 1.0 \\
\hline No. 4 & 7.85 & 43.30 & -5.00 & 0.8 & 7.81 & 43.25 & -5.12 & 0.9 \\
\hline No. 5 & 8.63 & 39.12 & -15.00 & 0.6 & 8.41 & 40.12 & -16.00 & 0.5 \\
\hline
\end{tabular}
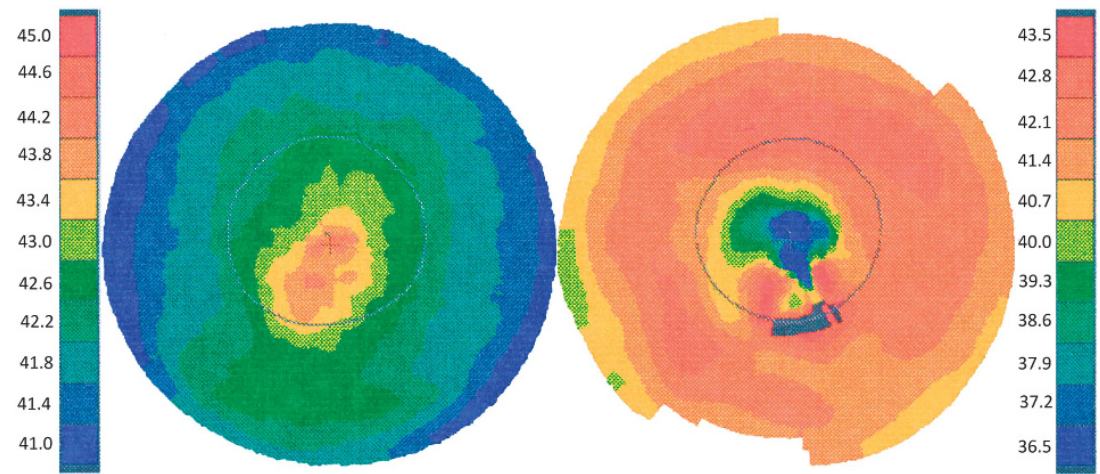

Figure 3 Topographic maps (in diopters) of left cornea of subject 5 pre-treatment (left) and one day post-treatment (right) with their corresponding color key.

diameter and refractive power. ${ }^{13}$ The rabbits tolerated the treatment well with topical anesthetic eye drops only. No injury was observed to the epithelium or Descemet layers with the slit lamp. The retinas also retained their normal appearance as seen with a direct and indirect ophthalmoscope. Histological analysis of the corneas did not evidence any Descemet tears, break in the collagen fibers, or any remarkable alteration. Supplementary Figure $\mathrm{B}$ is a histological slide of a corneal cross section of rabbit 8 .

The treated human eyes evidenced no significant corneal epithelium damage, no increase in intraocular pressure, no decrease in corrected visual acuity and normal slit lamp appearance of the cornea and anterior chamber with no increased cell and flare. Ophthalmoscopy did not evidence change or abnormality and pupillary reflex was present. As the vacuum was increased up to $760 \mathrm{~mm}-\mathrm{Hg}$, the eyes were examined again. This time we included Optical Coherence
Tomography (OCT) to provide some objective data of potential optic nerve and macula changes. None was observed. Eyes treated at full vacuum showed similar tolerance to the treatment and changes in corneal curvature. Two eyes of about $-3 \mathrm{D}$ flattened about $0.5 \mathrm{D}$. Two eyes of about $-6 \mathrm{D}$ flattened $1 \mathrm{D}$ and $1.5 \mathrm{D}$. A highly myopic eye of $-20 \mathrm{D}$ flattened $5 \mathrm{D}$. The flattening was demonstrated by the corneal topography and by subjective and objective refraction methods. The curvature of the treated corneas displayed the same revolution regularity as before treatment. The topographical maps showed less curvature in the treated central cornea with increasing and then decreasing curvature towards the periphery. Astigmatism remained essentially unchanged in all subjects. Spherical refractions remained spherical. Figure 3 is a pair of representative topographic maps of the cornea of subject 5 before and after treatment. The flattening of the human corneas 
remained unchanged 2 months after treatment. Human eyes have been followed for 2 years, showing little or no regression in their subjective and objective refraction. Corrected distance visual acuity (CDVA) in the decimal scale ranged between 0.3 and 1.5 before treatment and 0.5 and 2 after treatment. CDVA was equal or better after treatment for every eye. See Tables $1 c$ and $d$, that list the mean radii and power, spherical equivalent refraction, and CDVA for all subjects.

The corneas were examined pre and post-treatment with a slit lamp and CLSM, which allowed visualization of corneal cells. The cornea thickness was also measured with a standard ultrasonic pachymeter. The eyes had a normal appearance when tested $24 \mathrm{~h}$ after treatment. The collagen fibers of the stroma appeared transparent and intact after treatment. CLSM revealed no changes in shape of endothelial cells, characteristic of dead cells. Supplementary Figure C is a CLSM image of the endothelial cells of subject 2 one day post-treatment. There was no significant change in cell density after 2 years. The thickness of the cornea was reduced by an average of $7 \%$. The intraocular pressure pre-treatment and post-treatment was within normal range in all cases and no trend was present.

\section{Discussion}

The human and rabbit cornea are visco-elastic materials that continually deform or creep when strained beyond the elastic range. Such a deformation has been observed in strips of human and rabbit cornea in vitro. ${ }^{12}$ The rabbit and human cornea have substantial similarities in their elastic-plastic characteristics. 5,12 Anecdotal evidence such as permanent refractive anomalies after forceps delivery suggested that the human cornea can undergo plastic deformation. ${ }^{14}$

Stressing the cornea locally by the application of a vacuum produces a permanent deformation of the collagen fibers of the living cornea. This is the first time that collagen fibers have been permanently altered in vivo in a controlled manner. Numerous models have been developed to account for corneal deformation after refractive procedures, but they do not extend to plastic deformation. ${ }^{15}$ The corneal deformation described here changes corneal curvature, and therefore eye refraction. All corneas flattened and myopia was reduced in all human subjects. The extent of flattening of the cornea can be controlled by the shape and number of the device chambers, the level of vacuum, the duration and repetition of the procedure and the temperature, alone or in combination.

The corneal temperature is around $35^{\circ} \mathrm{C}$ at $25^{\circ} \mathrm{C}$ room temperature. By adjusting ambient temperature from 40 to $80^{\circ} \mathrm{C}$, the corneal temperature will vary from 37 to
$42{ }^{\circ} \mathrm{C} .{ }^{16,17}$ For a given plastic strain of the collagen fibers in the rabbit sclera, the higher the temperature, the greater the strain. ${ }^{18}$ The rabbit sclera at $39.5^{\circ} \mathrm{C}$ permanently strains $1.5 \%$ with a stress pressure of only $100 \mathrm{~mm} \mathrm{Hg}$. ${ }^{19}$ Assuming that the human cornea behaves the same way, elevating the temperature of the cornea could be an easy way to increase and control the flattening effect and the myopia corrected.

The reduction in the thickness of the central cornea was not unexpected. The cornea (as most materials) thins when strained. This effect can be permanent and has been observed even at near physiological intraocular pressures. ${ }^{20}$ Stressed human corneas have a Poisson ratio of about 0.5 ; their thickness reduces by $50 \%$ of what they strain. ${ }^{21}$ The central cornea strained radially as a result of peripheral vacuum stress in that direction because the central cornea and limbus were opposing that stress. Although it is undesirable that the cornea strains beyond the elastic range in the radial direction, it is unavoidable with a few large sub-chambers, needed for increased stress.

Myopia affects about one quarter of the western adult population, and more than one half of the population in some Asian countries. ${ }^{22}$ Myopia appears to be a feedback process amenable to control. ${ }^{23}$ Preventive methods are rarely used, and depend on the application of accurate models of myopia. Several surgical methods have been tried to correct myopia, but none is simple, or free of risks. Current surgical methods to correct myopia achieve their goal by invasively modifying the corneal curvature. Methods which are used, have been used or are being developed include: radial keratotomy ${ }^{24}$ (radial incisions made in the periphery of the cornea), excimer laser photorefractive keratectomy (PRK) ${ }^{25,26}$ (laser ablation of the anterior surface of the cornea), laser assisted in situ keratomileusis or (LASIK), ${ }^{27}$ Small Incision Lenticule Extraction (SMILE), implantation of intra ocular ring or lens ${ }^{28}$ and potentially, customized Corneal Collagen crosslinking (CXL). We call this new procedure 'Pneumatic Keratology.' The vacuum force flattens the central part of the cornea by distending the stressed areas where vacuum is applied, in a similar manner as radial keratotomy incisions extend the cornea perpendicularly to the length of the incisions. ${ }^{24}$

The duration and pressure applied corrected a fraction of the human subjects' myopia, up to $5 \mathrm{D}$ in one case, and close to 1 diopter (average $0.73 \mathrm{D}$ ) in the rabbits. The flattening of rabbits' corneas regressed to half a diopter three months later, and remained stable thereafter. The plastic property of the cornea could be used to correct not only myopia, but hyperopia and other refractive conditions. Our experiments show that the same vacuum force has different effects depending on the degree of the subject's myopia. The implication is that the higher the 
myopia, the less the ocular rigidity. Researchers have hypothesized that myopes have a weaker sclera prone to elongation. ${ }^{29}$ The weakened sclera of the rabbit exhibited a continuous elongation. ${ }^{19}$ Although our experiments were done on the cornea, it is known that sclera and cornea are very similar anatomically and mechanically. ${ }^{21,29-31}$ Our results, therefore, support the view that myopic eyes have weaker corneoscleral envelopes, and that their weakness is proportional to their myopia.

The way we produced a flattening of the cornea by the application of a vacuum force was by using openings elongated in the radial direction. An elongated opening produces a bulging of the cornea when the vacuum is applied, as depicted in Figure 4. The curvature of the deformed cornea in the elongated direction is necessarily less than in the perpendicular, short direction because $\alpha<\beta$. If the curved sections of the deformed cornea in the elongated and short directions in Figure 4 are approximated by circular segments, it is evident that the short direction strains more than the long direction. If the elongated openings are arranged radially, as with the device described here, the cornea can be made to strain elastically in the radial direction, while plastically in the circumferential direction. The chambers produced a bulging of the cornea not in the center but in the periphery. That is the result intended and achieved here with the device. The foregoing analysis is consistent with the analysis of stress in the spheroidal sclera. Greene reported that stress in the direction of the higher curvature in the equator of the sclera is greater than in the direction with less curvature. ${ }^{32}$ Although we preferred elongated openings, other openings also produce a net flattening of the cornea, as we have confirmed experimentally. Treatment of cadaver eyes with different openings and the variations in procedure described above has demonstrated greater flattening of the cornea that corrects up to $8 \mathrm{D}$ of myopia. These experiments will be published in a separate report when completed.

The maximum vacuum that can be applied to the eye is limited by the pressure outside the eye, normally the

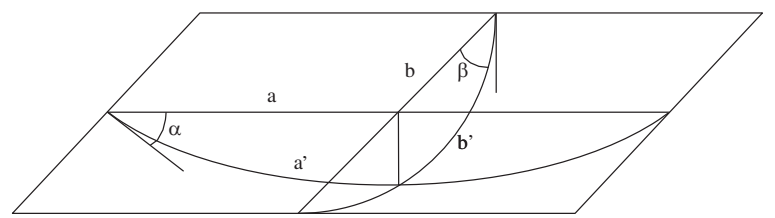

Figure 4 The strain (b'-b)/b caused by an elongated opening in the short direction of length $b$ is greater than the strain $\left(a^{\prime}-a\right) / a$ in the long direction $a$. Since the curvature of the arc of length $b^{\prime}$ is greater than the arc of length a' (because $a>b$ ), then $b^{\prime} / b>a^{\prime} / a$, which implies that $\left(b^{\prime}-b\right) / b>\left(a^{\prime}-a\right) / a$. atmospheric pressure, or about $760 \mathrm{~mm}-\mathrm{Hg}$. We have shown that such a pressure is sufficient to plastically deform the human cornea. In vitro data from Hoeltzel et al ${ }^{5}$ confirms that the rabbit cornea subjected to an equivalent intraocular pressure of about $760 \mathrm{~mm}-\mathrm{Hg}$ permanently strains by $6 \%$, while the human cornea subjected to only $400 \mathrm{~mm}-\mathrm{Hg}$ permanently strains by about $1 \%$. Permanent strain of the human cornea in vitro has been observed even under physiological range stress. ${ }^{20}$

\section{Conclusions}

The plastic, and therefore permanent, deformation of the cornea was obtained by straining the cornea with a vacuum force. A vacuum chamber with radial openings flattens the cornea. A flatter cornea corrects or reduces myopia.

The ability to modify collagen tissue in a controlled manner has implications in organs beyond the eye. Tendons, cartilage, heart valves and blood vessels for example, conceivably could be custom modified for corrective purposes.

\section{Summary}

\section{What was known before}

- The eyeball, and in particular the cornea, deforms in vitro by the application of a distending force like other viscoelastic tissue. If the force is large enough, the cornea strains beyond the elastic range, and a permanent deformation occurs. Such permanent strain is referred to as 'plastic' strain. The phenomenon however has never been observed or produced on living tissue. This report seeks to demonstrate that the central radius of a patient's cornea can be altered, in a controlled manner designed to correct refractive errors.

What this study adds

- The living cornea has been permanently deformed for the first time with an external force. The method described here can be used to flatten the cornea by non-invasive means. A flatter cornea corrects or reduces myopia.

\section{Conflict of interest}

The author declare no conflict of interest.

\section{Acknowledgements}

We are especially grateful to the Hospital Provincial, Valencia, Hospital de la Cruz Roja, Barcelona, Hospital Provincial, Castellon, Spain and the Centro de Microcirugia Ocular y Laser, Santo Domingo, Dominican Republic. The expert assistance of doctors Enrique Fariza, Cesar Villa, Victor Menezo, Jorge Lopez, Donald Sanders, David Brown, Del Valle, Rafael Feliz, Carlos Jimenez and Jordi Desola propelled this work. Further appreciation to 
Mary Goon for her writing assistance. This work was done at nonprofit institutions with no proprietary rights or interest. The author expects that other parties will use the results here to further develop and bring this technology to the market.

\section{References}

1 Boyce BL, Jones RE, Nguyen TD, Grazier JM. Stress-controlled viscoelastic tensile response of bovine cornea. J Biomech 2007; 40: 2367-2376.

2 Elsheikh A, Anderson K. Comparative study of corneal strip extensiometry and inflation tests. J R Soc Interface 2005; 2: 177-185.

3 Nguyen TD, Jones RE, Boyce BL. A nonlinear anisotropic viscoelastic model for the tensile behavior of the corneal stroma. J Biomech Eng 2008; 130: 041020.

4 Hatami-Marbini H, Rahimi A. Effects of bathing solution on tensile properties of the cornea. Exp Eye Res 2014; 120: 103-108.

5 Hoeltzel DA, Alman P, Buzard K, Choe K. Strip extensiometry for comparison of the mechanical response of bovine, rabbit, and human corneas. J Biomech Eng 1992; 114: 202-215.

6 Boschetti F, Triaca V, Spinelli L, Pandolfi A. Mechanical characterization of porcine corneas. J Biomech Eng 2012; 134: 031003.

7 Boyce BL, Grazier JM, Jones RE, Nguyen TD. Full-field deformation of bovine cornea under constrained inflation conditions. Biomaterials 2008; 29: 3896-3904.

8 Elsheikh A, Wang D, Pye D. Determination of the modulus of elasticity of the human cornea. J Refract Surg 2007; 23: 808-818.

9 Elsheikh A, Wang D, Rama P, Campanelli M. Experimental assessment of human corneal hysteresis. Curr Eye Res 2008; 33: 205-213.

10 Hennighausen H, Feldma ST, Bille JF, McCulloch AD. Anterior-posterior strain variation in normally hydrated and swollen rabbit cornea. Invest Ophthalmol Vis Sci 1998; 39: 253-262.

11 Shin TJ, Vito RP, Johnson LW, McCarey BE. The distribution of strain in the human cornea. J Biomech 1997; 30: 497-503.

12 Jue B, Maurice DM. The mechanical properties of the rabbit and human cornea. J Biomech 1986; 19: 847-853.

13 Rowsey JJ, Balyeat HD, Monlux R, Holladay J, Waring GO, Lynn MJ. Prospective evaluation of radial keratotomy. Ophthalmology 1988; 95: 322-334.

14 Angel LK, Rob RM, Berson FG. Visual prognosis in patients with ruptures in Descement's membrane due to forceps injury. Arch Ophthalmol 1981; 99: 2137.

15 Hamed Sayed AA, Solouma NH, El-Berry AA, Kadah YM. Finite element models for computer simulation of intrastromal photorefractive keratectomy. J Mech Med Biol 2011; 11: 1255-1270.

16 Slettedal JK, Ringvold A. Correlation between corneal and ambient temperature with particular focus on polar conditions. Acta Ophthalmol 2015; 93(5): 422-426.

17 Fabiani C, Li Voti R, Rusciano D, Mutolo MG, Pescosolido N. Relationship between corneal temperature and intraocular pressure in healthy individuals: a clinical thermographic analysis. J Ophthalmol 2016; 2016: 3076031.

18 Greene PR, McMahon TA. Scleral creep vs. temperature and pressure in vitro. Exp Eye Res 1979; 29: 527-537.

$19 \mathrm{Ku}$ DN, Greene PR. Scleral creep in vitro resulting from cyclic preasure pulses: applications to myopia. Am J Optom Physiol Opt 1981; 58: 528-535.

20 Lombardo G, Serrao S, Rosati M, Lombardo M. Analysis of the viscoelastic properties of the human cornea using scheimpflug imaging in inflation experiment of eye globes. PLoS One 2014; 9: e112169.

21 Uchio E, Ohno S, Kudoh J, Aoki K, Kisielewicz LT. Simulation model of an eyeball based on finite element analysis on a supercomputer. Br J Ophthalmol 1999; 83: 1106-1111.

22 Ward B, Thompson FB. The Myopias: Pathogenesis and Pathophysiology. In Myopia Surgery. Macmillan Publishing: New York, NY, USA, 1990; 1-30.

23 Medina A. The progression of corrected myopia. Graefes Arch Clin Exp Ophthalmol 2015; 253: 1273-1277.

24 Fyodorov SN, Durnev VV. Operation of dosage dissection of corneal circular ligament in cases of myopia of mild degree. Ann Ophthalmol 1979; 11: 1885-1890.

25 Trokel ST, Srinivasan R, Braren B. Excimer laser treatment on the cornea. Am J Ophthalmol 1983; 96: 710.

26 Gartry D, Muir MK, Marshall J. Excimer laser treatment of corneal surface pathology: a laboratory and clinical study. Br J Ohthalmol 1991; 75: 258-265.

27 Pallikaris IG, Papatzanaki ME, Siganos DS, Tsilimbaris MK. A corneal flap technique for laser in situ keratomileusis. Human studies. Arch Ophthalmol 1991; 109: 1699-1702.

28 AlSamman AH. Anterior chamber foldable phakic intra ocular lens safety and efficacy. J Clin Res Ophthalmol 2016; 3(1): 011-017.

29 Curtin BJ. Physiolpathologic aspects of scleral stress-strain. Trans Am Ophthalmol Soc 1969; 67: 417-461.

30 Gloster J, Perkins ES, Pommier ML. Extensibility of strips of sclera and cornea. Br J Ophthalmol 1957; 41: 103-110.

31 Woo SL, Kobayashi AS, Schlegel WA, Lawrence C. Non linear material properties of intact cornea and sclera. Exp Eye Res 1972; 14: 29-39.

32 Greene PR. Stress amplification and plastic flow for spheroidal shells: Applications to myopia. Rheol Acta 1987; 26: $479-484$.

Supplementary Information accompanies this paper on Eye website (http://www.nature.com/eye) 